\title{
Formación de Profesores de Lengua Portuguesa en la Perspectiva de la Educación de Sordos ${ }^{1}$
}

\author{
Portuguese Language Teachers' Education on the Perspective of \\ EDUCATION OF THE DEAF
}

\author{
Raquel Schwenck de Mello Vianna SOARES 2 \\ Wellington Danilo SOARES ${ }^{3}$ \\ Daniel Antunes FREITAS ${ }^{4}$ \\ Ludmila Cotrim FAGUNDES ${ }^{5}$ \\ André Augusto Dias SILVEIRA ${ }^{6}$ \\ Bárbara Carvalho FERREIRA ${ }^{7}$
}

\begin{abstract}
RESUMEN: Las dificultades de las personas sordas en la lengua portuguesa escrita y del docente en su enseńanza, sumadas a la escasez de investigaciones en el área, hacen relevante la revisión de estrategias en el proceso de formación docente. Se trata de una investigación cualitativa y cuantitativa con 16 profesores de los últimos años de Educación Primaria y Secundaria de la lengua portuguesa de la red de escuelas públicas que trabajaron con estudiantes Sordos en algún momento de su experiencia docente. Fueron seleccionados 8 profesores de la Educación Inclusiva y 8 de una escuela Bilingüe para Sordos. Se utilizó el software NVivo10 para el análisis de datos. En la formación inicial, los docentes no tuvieron contacto con la Lengua de Seńas ni estrategias metodológicas específicas para la enseńanza de Sordos. En la educación continua, la enseńanza más eficaz fue la de otros profesores con más experiencia. Destaca una dicotomía entre los profesores de las escuelas inclusivas y los de la escuela bilingüe para Sordos, donde los primeros utilizam la Lengua Brasileña de Señales - Libras como una lengua accesoria para la enseńanza del portugués escrito, ya, los docentes de la escuela bilíngue la utilizan como lengua de instrucción con el uso del metalenguaje. En cuanto a las concepciones, los docentes sugirieron algunas prácticas en formación para trabajar con el público Sordo. Por tanto, es fundamental invertir en escuelas inclusivas y bilingües que trabajen con estudiantes Sordos, así como en la formación continua de los docentes, proponiendo grupos de estudio con todo el equipo pedagógico como parte del proceso de formación.
\end{abstract}

PALABRAS CLAVE: Formación de Profesores. Lengua Portuguesa. Educación de Sordos. Lengua Brasileña de Señas.

ABSTRACT: The difficulties of the deaf in written Portuguese and of the teacher in teaching, added to the scarcity of research in the area, make the revision of strategies in the process of teacher training relevant. This is a qualitative and quantitative research

\footnotetext{
${ }^{1}$ https://doi.org/10.1590/1980-54702021v27e0004

${ }^{2}$ Doutora em Educação pela Universidad Catolica de Santa Fe - Argentina. Docente da Universidade Federal dos vales do Jequitinhonha e Mucuri - UFVJM. Diamantina / Minas Gerais / Brasil. E-mail: raquel.schwenck@ufvjm.edu.br. ORCID: https://orcid. org/0000-0003-2298-6109

${ }^{3}$ Doutor em Ciências da Saúde pela Universidade Estadual de Montes Claros - Unimontes, docente da Universidade Estadual de Montes Claros - Unimontes. Montes Claros / Minas Gerais / Brasil. E-mail: wdansoa@yahoo.com.br. ORCID: https://orcid. org/0000-0001-8952-9717

${ }^{4}$ Doutor em Ciências da Saúde pela Universidade Estadual de Montes Claros - Unimontes. Docente do curso de Medicina da Universidade Estadual de Montes Claros - Unimontes. Montes Claros / Minas Gerais / Brasil. E-mail: danielmestradounincor@ yahoo.com.br. ORCID: https://orcid.org/0000-0001-7023-8610

${ }^{5}$ Acadêmica do curso de Medicina da Universidade Estadual de Montes Claros - Unimontes. Bolsista do Programa Institucional de Bolsas de Iniciação Científica. Montes Claros / Minas Gerais / Brasil. E-mail: ludmilacotrimfagundes@gmail.com. ORCID: http://orcid.org/0000-0001-5841-8280

${ }^{6}$ Acadêmico do curso de Medicina da Universidade Estadual de Montes Claros - Unimontes. Bolsista do Programa Institucional de Bolsas de Iniciação Científica. Montes Claros / Minas Gerais / Brasil. E-mail: andreadsilveiran@gmail.com. ORCID: https:// orcid.org/0000-0002-2082-3288

${ }^{7}$ Doutora em Psicologia pela Universidade Federal de São Carlos - UFSCar. Docente da Universidade Federal dos Vales do Jequitinhonha e Mucuri - UFVJM. Diamantina / Minas Gerais / Brasil. E-mail: babipocos@yahoo.com.br. ORCID: https://orcid. org/0000-0002-9759-5682
} 
with 16 teachers from the last years of Elementary and High School of Portuguese language in the public-school network who worked with deaf students at some point in their teaching experience. Eight teachers from Inclusive Education and eight from a Bilingual School for the Deaf were selected. NVivo10 software was used for data analysis. In the initial training, the teachers did not have contact with Sign Language or specific methodological strategies for teaching the Deaf. In continuing education, the most effective teaching was that of other, more experienced teachers. There is a dichotomy between teachers from inclusive schools and those from the bilingual school for the deaf, where the former use the Brazilian Sign Language as an accessory language for the teaching of written Portuguese, whereas teachers in the bilingual school use it as the language of instruction with the use of metalanguage. Regarding the conceptions, the teachers suggested some training practices to work with the Deaf audience. Therefore, it is essential to invest in inclusive and bilingual schools that serve deaf students, as well as in the continued training of teachers, proposing study groups with the entire pedagogical team as part of the training process.

KEYWORDS: Teacher Training. Portuguese Language. Deaf Education. Brazilian Sign Language.

\section{INTRODUÇCIÓN}

La disciplina de Lengua Brasileña de Señas (Libras) se ha vuelto obligatoria en los cursos de formación docente para el ejercicio de la docencia en Brasil en 2005, en los niveles secundario y superior. Las escuelas pueden adoptar un carácter bilingüe o inclusivo.

La metodología del bilingüismo para la educación de Sordos funciona a través de dos enfoques, el visual-espacio, en Libras; y la modalidad escrita del portugués (Vieira \& Molina, 2018). Esta metodología está respaldada por la Ley n ${ }^{\circ} 10436$ (2002) y el Decreto n 5626 (2005) (Kumada \& Prieto, 2019).

La inclusión escolar, por su parte, se basa en la introducción del individuo Sordo ${ }^{8}$ en las escuelas ordinarias con propuestas inclusivas, de las más variadas metodologías (Morais \& Martins, 2020), esto lo garantiza la Política Nacional de Educación Especial, desde la perspectiva de la educación inclusiva y la Ley n ${ }^{\circ} 13146$ (2015) (Kumada \& Prieto, 2019).

En las dos perspectivas de educación para Sordos, la Lengua Portuguesa en la modalidad escrita debe ser presentada como segunda lengua (L2). De acuerdo Silva (2017), el portugués como L2 para Sordos debe ser enseñado utilizando metodologías específicas, considerando las especificidades en varios aspectos, sea lingüísticos, culturales, educacionales, etc.

Importante explicitar que la educación de Sordos se da en la Lengua de Señas, siendo esta reconocida como medio de comunicación, constitución del sujeto y concepción del mundo. Sin embargo, el idioma oral es reconocido como su segunda lengua en la modalidad escrita (Oliveira, 2018).

Tratándose de un aprendizaje de una lengua intermodal (dos modalidades distintas: Libras de modalidad visual-espacial y Lengua Portuguesa de modalidad oral-auditiva) no es difícil encontrar textos escritos por Sordos con características de la Lengua de Señas (Brochado, 2003; Quadros \& Schmiedt, 2006). Basándose en eso, es necesario adaptar una didáctica de enseñanza que lleve a cabo las especificidades del aprendiz Sordo (Bernieri-Souza \& Vasconcelos, 2021).

A pesar de las iniciativas de formación de Sordos, existe un gran obstáculo, el déficit en la formación y calificación de los docentes de escuelas inclusivas y bilingües, especialmente al analizar a los docentes que trabajan en los primeros años de escolaridad. Esto se demuestra en

\footnotetext{
${ }^{8}$ En este estudio, el término Sordo será utilizado con la inicial $S$ mayúscula, pues adopta concepto de autores que analizan el Sordo bajo la perspectiva de la identidad, cultural y lingüística, con sus experiencias basadas en el aspecto visual (Sacks, 2010; Sánchez, 1990).
} 
los planes de estudio de la enseñanza, donde se carece de conocimientos sobre el aprendizaje de los Sordos, y este conocimiento suele depender del autoaprendizaje del docente en formación y de su formación continua (Calixto et al., 2019).

Para esto, el estudio tiene como objetivo analizar la capacitación de profesores de Lengua Portuguesa de escuelas inclusivas y bilingües y la práctica con alumnos Sordos.

\section{Método}

Esta es una investigación cuali-cuantivativa que tiene como instrumento la entrevista semiestructurada y el proceso de análisis basado en el Análisis de Contenido (AC) de Bardin (2011), utilizándose como herramienta de análisis el software NVivo 10.

El Análisis del Contenido (AC) es "un conjunto de técnicas de análisis de las comunicaciones intentando obtener, por medio de procedimientos sistemáticos y objetivos de descripción del contenidos de los mensajes, indicadores (cuantitativos o no) que permitan la inferencia de conocimientos relacionados a las condiciones de producción/recepción (variables inferidas) de esos mensajes", permite tratar los datos colectados al hacer una interpretación siguiendo criterios metodológicos rigurosos, no solo utilizando la intuición o interpretación sin criterios (Bardin, 2011, p. 42).

En este estudio fue utilizado el software NVivo10 que es destinado al tratamiento de datos en investigación cualitativa. Con este software el investigador puede organizar, visualizar, interconectar y editar documentos, crear categorías, codificar, controlar, filtrar, hacer búsquedas y cuestionar los datos con los objetivos de contestar a las cuestiones de investigación. Entre otras ventajas, se confirma la coherencia de la codificación, además de permitir una investigación a nivel científico, regida por pautas fácticas, desprovista de opiniones infundadas (Lage, 2011).

Participaron de la investigación 16 docentes de Lengua Portuguesa de la red pública de enseñanza que impartían clases para alumnos Sordos en algún momento de su experiencia docente. De estos 16, ocho pertenecían a escuelas estatales inclusivas del Estado de Minas Gerais, los otros ocho a una escuela federal bilingüe para Sordos del Estado del Rio de Janeiro. Para esto, se seleccionaron profesores de las series iniciales de la Enseñanza Fundamental y profesores de la Enseńanza Media.

Para este estudio se elaboró un Guión de Entrevista Semiestructurado compuesto por 40 preguntas sobre: la capacitación inicial y continua del docente para actuación en la perspectiva de la Educación Inclusiva y Bilingüe de alumnos Sordos, metodologías utilizadas por estos profesores en la enseńanza de Lengua Portuguesa para Sordos y sugerencias sobre cómo optimizar sus prácticas.

En el horario y lugar firmados para realización de las entrevistas se presentaba al profesor el "Termo de Consentimento Livre Esclarecido" (TCLE). La entrevista fue realizada individualmente y grabada en audio en un aula de clase de una escuela pública en el horario de intervalo entre las clases para que no hubiese interferencia externa o perjuicio al trabajo del docente. Todas las entrevistas fueron realizadas en las propias escuelas, en horario disponible para el profesor o en post-horario, habiendo sido realizadas en aulas vacías, salón de profesores 
o biblioteca. Tras la realización de las entrevistas, estas fueron transcriptas integralmente por la investigadora para que no se perdiese contenido, y con el propósito de análisis posterior.

El proyecto fue aprobado por al Comité de Ética en Pesquisa de las Facultades Unidas del Norte de Minas - Funorte, parecer no 1.274.516, además de haber sido orientado por el Consejo Nacional de Salud bajo la Resolución no 466/12.

Las entrevistas fueron grabadas en audio y después transcriptas integralmente para que no perder contenido, y con el propósito de análisis posterior.

Las entrevistas fueron tratadas a partir de las transcripciones, por medio del software NVivo10, siguiendo la metodología del Análisis de Contenido y centrándose en los objetivos de la investigación para la generación de informes con datos cuantitativos, como frecuencias de codificación de las categorías por participante, frecuencia de codificación por categoría, y datos cualitativos por medio de la ejemplificación con las palabras de los entrevistados, concluyendo el análisis en la construcción de un árbol categorial final.

Para la construcción de este árbol categorial fueron necesarios los siguientes pasos: pre-análisis de las entrevistas a través de la lecturas de las mismas sin aspirar a los objetivos propuestos, limitándose solo en las palabras y contenidos expuestos por los docentes, denominándose este paso como lectura flotante del contenido. Comparación de los datos de las entrevistas con los objetivos de la investigación, ateniéndose a las palabras más expresivas y recurrentes. Propuesta inicial de las categorías (árbol categorial de base). Lectura con criterio y selección de fragmentos que combinaban con las categorías preestablecidas, necesitándose ańadir, sacar y reorganizar las categorías, con la culminación en el árbol categorial final tratamiento de los datos a partir de la organización de las palabras de los entrevistados en categorías a las cuales se adecuaban al contenido según los temas y, al final, adecuación del árbol por medio de la eliminación, colocación y reorganización de las categorías.

El tratamiento de los datos fue realizado, simultáneamente, por tres investigadores. Al final, el material fue analizado en conjunto por los tres, sirviendo de contribución fundamental de la normalización de los resultados. Los individuos tenían total conocimiento de las herramientas de trabajo y experiencia a nivel del análisis estadístico de contenido, utilizándose instrumentos informáticos.

Se elaboraron informes del proyecto (todos los posibles, a modo de jerarquía) y de las fuentes para verificación de la evolución del trabajo tras la importación de los documentos. Fueron aún exportadas para documentos de procesamiento de texto.

Al paso que era tratado el material fueron elaborados modelos dinámicos de correlación entre ítems, y que eran transformados en modelos estáticos del trabajo. Los modelos estáticos permiten verificar el material existente en cada momento, pues no se alteran en función del material que estaba adyacente a ellos, haciendo posible, así, averiguar la evolución del trabajo a lo largo de su ejecución.

Enseguida, fueron elaboradas agregaciones-tipo, buscando, en este momento, patrones, regularidades, y también contrastes y singularidades. Esta fase es igualmente caracterizada por un ejercicio de fundamentación de las decisiones hechas, lo que se consolida en un trabajo 
de constate contextualización y de referencia de los extractos frente a cada fuente documental. La constitución de agregaciones-tipo fue orientada por el presumido de la comparabilidad, es decir, la agrupación o distinción de los programas, con perfiles idénticos y distintos.

El árbol categorial final está presentado con tres ramas (obtención de conocimiento, metodologías de enseńanza de PL2 y variables de eficacia pedagógica) que se dividieron en nueve categorías (no formal, capacitación de profesores, estrategias de realización, acción-reflexión-acción, comunicación directa, ausencia de trabajo diferenciado, transferencias de responsabilidad para el intérprete, concienciación sobre la importancia de buenas prácticas y estrategias de perfeccionamiento de las prácticas) y siete subcategorías (inicial, continua, cursos libres, ausencia de UC's específicas sobre Sordos en la capacitación inicial u continua y existencia de UC's específicas sobre Sordos en la capacitación inicial y continua). Se aclara que las ramas, categorías y subcategorías se relaciona al portugués como segunda lengua para Sordos y todas con la finalidad de contestar a los objetivos propuestos por la investigación.

\section{Resultados}

Se observa, a través de la Tabla 1, que todos los participantes son graduados en Letras, en su mayoría en portugués, siendo tres en Letras Inglés, de estos, dos docentes de las escuelas inclusivas y uno de la escuela bilingüe para Sordos. Del total, se destaca tres que solo poseen graduación y que estos son de escuelas inclusivas. Por otro lado, con relación a los docentes con nivel de titulación más elevado, hay tres con Maestría y cuatro Doctores, todos pertenecen a la escuela bilingüe para Sordos. Se hace relevante especificar que los participantes E2 a E9 son docentes pertenecientes a escuelas inclusivas, mientras que E10 al E17 son docentes de la escuela bilingüe para Sordos.

Con relación al tiempo de capacitación de los docentes entrevistados, es posible verificar que los profesores de las escuelas inclusivas de esta investigación, de promedio, están graduados hace más tiempo que los de la escuela bilingüe para Sordos, aunque aquellos tengan menor nivel de titulación que estos.

Además, es posible observar que, en lo que dice respecto al nivel de conocimiento que los profesores entrevistados evalúan poseer en Lengua Brasileña de Señas, se puede constatar que cuatro juzgan no poseer ningún tipo de conocimiento en la lengua, todos estos pertenecen a escuelas inclusivas, representando mitad de los entrevistados con este perfil; tres juzgan tener conocimiento avanzado y uno fluente en Libras, todos esos docentes de la escuela bilingüe para Sordos.

Sobre los cursos de capacitación continua, los docentes entrevistados de las escuelas inclusivas representan la mayoría de los docentes sin ningún curso, en un total de cinco de estos. En la perspectiva de los docentes entrevistados de la escuela bilingüe para Sordos, solo tres no cursaron ningún tipo de capacitación continua. De modo más específico en con respecto a la capacitación en Libras, de los docentes de las escuelas inclusivas, apenas uno cursó Libras básico; no obstante, cuatro docentes de los de la escuela bilingüe para Sordos cursaron Libras. Es importante resaltar que esta capacitación en Libras es ofrecida gratuitamente como parte de la capacitación docente de la escuela bilingüe para Sordos, como requisito para el trabajo con 
este público, por otro lado en las escuelas inclusivas esto no es requisito o un curso ofrecido gratuita o periódicamente.

\section{Tabla 1}

Caracterización de los participantes en términos de capacitación inicial y continua

\begin{tabular}{l|l|l|l|l|l}
\hline Participante & $\begin{array}{l}\text { Capacitación } \\
\text { inicial }\end{array}$ & $\begin{array}{c}\text { Ańo de } \\
\text { graduación }\end{array}$ & \multicolumn{1}{|c}{$\begin{array}{c}\text { Más elevada } \\
\text { titulación }\end{array}$} & \multicolumn{1}{|c}{$\begin{array}{c}\text { Nivel en } \\
\text { Libras }\end{array}$} & $\begin{array}{c}\text { Capacitación con- } \\
\text { tinua en el área }\end{array}$ \\
\hline E2 & L. P. & 2005 & Especialización & Básico & Libras \\
\hline E3 & L. P. & 2004 & Graduación & Ninguno & Ninguna \\
\hline E4 & L. P. I. & 2003 & Especialización & Básico & PL2 \\
\hline E5 & L. P. & 1997 & Especialización & Básico & Ninguna \\
\hline E6 & L. P. I. & 1992 & Graduación & Ninguno & Ninguna \\
\hline E7 & L. P. & 2001 & Especialización & Básico & PL2 Sordos- \\
\hline E8 & L. P. & 2002 & Graduación & Ninguno & Ninguna \\
\hline E9 & L. P. & 2002 & Especialización & Ninguno & Ninguna \\
\hline E10 & L. P. & 2007 & Doctorado & Fluente & AEE Sordos - Libras \\
\hline E11 & L. P. L. & 2009 & Maestría & Intermediario & Libras \\
\hline E12 & L. P. I. & 2004 & Doctorado & Intermediario & Libras \\
\hline E13 & L. P. L. & 2007 & Maestría & Básico & Libras \\
\hline E14 & L. P. L. & 2000 & Maestría & Avanzado & Ninguna \\
\hline E15 & L. P. L. & 2000 & Doctorado & Avanzado & Ninguna \\
\hline E16 & L. P. L. & 2004 & Doctorado & Avanzado & Ninguna \\
\hline E17 & L. P. & 2005 & Especialización & Intermediario & Libras \\
\hline
\end{tabular}

Nota: L. P. = Letras Portugués; L. P. I.= Letras Portugués Inglés; L. P. L.= Letras Portugués Literatura; PL2

= Portugués como segunda lengua; C.T.T.I.L. = Curso Técnico en Traducción e Interpretación de Libras; AEE = Atendimiento Educacional Especializado.

Con la finalidad de representación visual y comprensión de la construcción de las categorías pertinentes a la investigación, se presenta el árbol categorial final, elaborado para este estudio, siguiendo criterios del Análisis de Contenido (Bardin, 2011) y utilizando como soporte el software NVivo10, considerando las palabras de los entrevistados y los objetivos propuestos en la investigación. Los datos referentes al árbol categoría final están presentados en la Figura 1. 


\section{Figura 1}

\section{Arbol categorial final a través del software NVivo10}

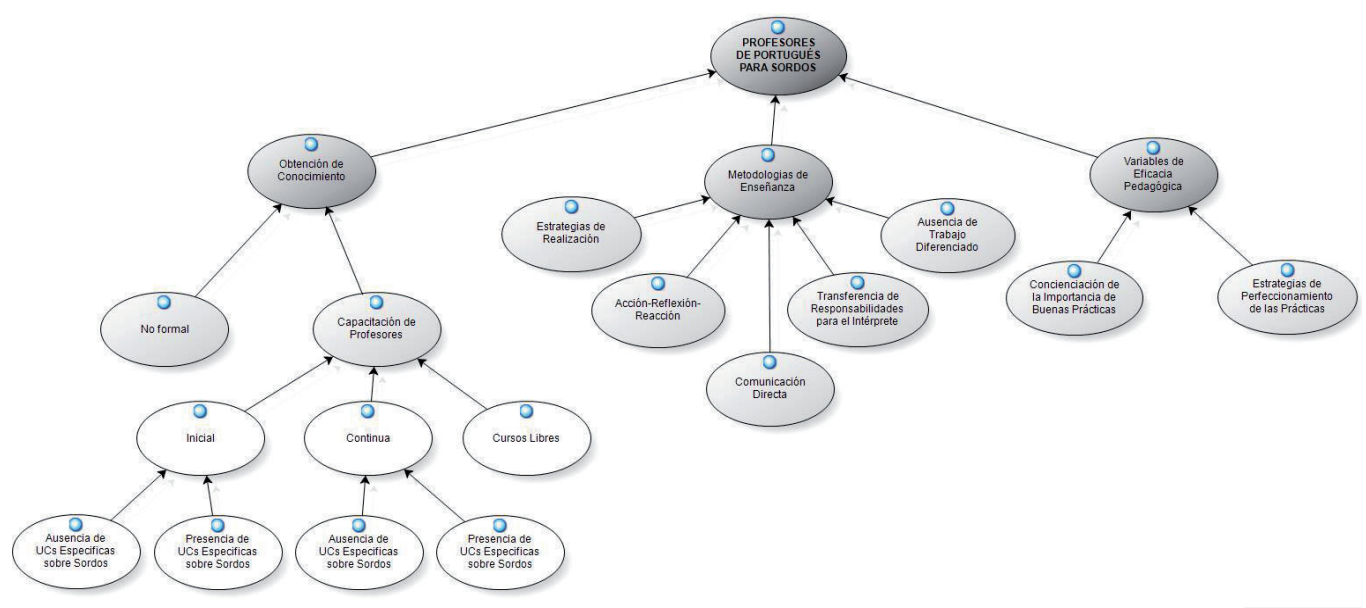

Pasando por los pasos de elaboración del árbol categorial (Figura 1), se hizo a la estructura final de tres ramas categoriales (obtención de conocimiento" metodologías de enseñanza de PL2 ${ }^{10}$ y variables de eficacia pedagógica ${ }^{11}$ ), subdivididos en nueve categorías (no formal ${ }^{12}$, capacitación de profesores ${ }^{13}$, estrategias de realización ${ }^{14}$, acción-reflexión-reacción ${ }^{15}$, comunicación directa $^{16}$, ausencia de trabajo diferenciado ${ }^{17}$, transferencia de responsabilidad para el intérprete ${ }^{18}$, concienciación de la importancia de buenas prácticas y estrategias de perfeccionamiento de las prácticas ${ }^{19}$ ) y siete subcategorías (inicial, continua, cursos libres, ausencia de UC's específicas sobre Sordos en la capacitación inicial y continua y existencia de UC's específicas sobre Sordos en la capacitación inicial y continua).

\footnotetext{
${ }_{9}^{9}$ Dice respecto a la forma como los profesores obtuvieron el conocimiento relacionado al PL2 para Sordos y a la Lengua Brasileńa de Señas.

${ }^{10}$ Relacionada con las estrategias utilizadas por los docentes para la enseñanza de Portugués como Segunda Lengua para Sordos

${ }^{11}$ Concienciación del docente con relación a las estrategias pedagógicas para una enseñanza eficaz y sugerencias de perfeccionamiento

${ }^{12}$ Cuando los profesores adquieren el conocimiento en el contacto con los propios alumnos Sordos, otros profesionales del ambiente escolar, o incluso por medio de los profesionales traductores/intérpretes de Libras, o sea, de manera no sistematizada, no intencional.

${ }^{13}$ Aprendizaje reglado, sistematizado, intencional, impartido por profesionales especializados, con reglamentación propia y programas institucionalizados.

${ }^{14}$ Estrategias metodológicas utilizadas por el docente en clase con el alumno Sordo.

${ }^{15}$ Actitudes del docentes en lo que dice respecto a la enseńanza de PL2 a los Sordos

${ }^{16}$ Comunicación del docente directamente con el alumno Sordo, o sea, sin interferencia o mediación del profesional traductor/ intérprete de Libras.

${ }^{17}$ Docente que no utiliza estrategias metodológicas diferenciadas para la enseñanza del PL2 a Sordos.

${ }^{18}$ Transferencia de la responsabilidad de enseńanza para otro profesional que logre comunicarse con su alumno.

${ }^{19}$ Valorización del docente con relación a buenas prácticas en la enseńanza de PL2 para Sordos.
} 
A partir de los datos de la Tabla 2, es posible deprender que de los profesores entrevistados, solamente dos, E13 y E14, docentes de la escuela bilingüe, tuvieron acceso a las asignaturas relacionadas a la Educación de Sordos, a nivel de postgrado. En este sentido, el entrevistado E16, docente de la escuela bilingüe para Sordos, fue el que menos mencionó la ausencia de unidades curriculares (UC's) específicas sobre Sordos en su capacitación inicial con tan solo 0,48\% de toda subcategoría, mientras el docente E2, de una escuela inclusiva, fue el que más hizo mención a esta ausencia con $24,66 \%$ de todos los entrevistado en esta subcategoría.

\section{Tabla 2}

Porcentaje de la declaración de los docentes con relación a la Capacitación inicial, continua e no formal de los docentes

\begin{tabular}{|c|c|c|c|c|c|c|}
\hline $\begin{array}{l}\text { Partici- } \\
\text { pante }\end{array}$ & $\begin{array}{c}\text { No } \\
\text { formal }\end{array}$ & $\begin{array}{l}\text { Ausencia de UC's } \\
\text { Especificas } \\
\text { sobre Sordos Ca- } \\
\text { pacitación Inicial }\end{array}$ & $\begin{array}{l}\text { Existencia de UC's } \\
\text { Especificas sobre } \\
\text { Sordos Capacitaci- } \\
\text { ón Inicial }\end{array}$ & $\begin{array}{c}\text { Ausencia de UC's } \\
\text { Especificas sobre } \\
\text { Sordos } \\
\text { Capacitación } \\
\text { Continua }\end{array}$ & $\begin{array}{l}\text { Existencia de UC's } \\
\text { Especificas } \\
\text { sobre Sordos Capa- } \\
\text { citación Continua }\end{array}$ & $\begin{array}{l}\text { Cursos } \\
\text { Libres }\end{array}$ \\
\hline E2 & 2,78 & 24,66 & 0 & 23,81 & 0 & 16,05 \\
\hline E3 & 3,97 & 4,6 & 0 & 0 & 0 & 0 \\
\hline E4 & 1,4 & 2,85 & 26,53 & 0 & 0 & 5,76 \\
\hline E5 & 7,72 & 3,73 & 20,18 & 0 & 0 & 3,41 \\
\hline E6 & 8,91 & 3,65 & 0 & 0 & 0 & 8,11 \\
\hline E7 & 9,38 & 4,76 & 9,3 & 19,58 & 0 & 4,53 \\
\hline E8 & 5,92 & 1,51 & 7,03 & 0 & 0 & 9,71 \\
\hline E9 & 11,59 & 3,73 & 4,54 & 0 & 0 & 0 \\
\hline E10 & 0,34 & 8,49 & 2,72 & 0 & 0 & 20,91 \\
\hline E11 & 6,3 & 16,65 & 12,24 & 28,57 & 0 & 4,11 \\
\hline E12 & 5,41 & 5,95 & 0 & 0 & 4,71 & 16,32 \\
\hline E13 & 6,36 & 2,14 & 0 & 0 & 19,87 & 1,28 \\
\hline E14 & 20,3 & 12,77 & 0 & 3,17 & 75,42 & 1,55 \\
\hline E15 & 1,76 & 1,43 & 0 & 5,29 & 0 & 5,12 \\
\hline E16 & 6,75 & 0,48 & 0 & 15,87 & 0 & 1,44 \\
\hline E17 & 1,11 & 2,62 & 17,46 & 3,7 & 0 & 1,71 \\
\hline
\end{tabular}

Desde el análisis de la Tabla 3, se observa que el conocimiento adquirido con la capacitación inicial, la capacitación continua y cursos libres por los docentes entrevistados puede estar siendo aplicado en la práctica docente con alumnos Sordos por medio de estrategias de realización, y el entrevistado E14, docente de la escuela bilingüe, es el que más utiliza estrategias específicas para la enseńanza de PL2 para Sordos, con 13,09\% de las referencias a esta subcategoría. Otro entrevistado que hizo mención a las estrategias de realización fue el E10, con $10,81 \%$ de toda esta subcategoría, un cuantitativo interesante, siendo el segundo docente más mencionó tales estrategias.

En las declaraciones de los entrevistados y por los datos cuantitativos presentados se observa que los docentes de la escuela bilingüe para Sordos son más reflexivos que los de las escuelas inclusivas, dado que los docentes que más se refirieron a esta subcategoría fue uno de 
la escuela bilingüe para Sordos (E14), mientras que el docente que menos se refirió fue el E3 (0\%) de una escuela inclusiva.

La Tabla 3 permite observar aún que la ausencia de trabajo diferenciado es un tema recurrente en las declaraciones de los docentes de las escuelas inclusivas, con una representación sustancial, el E3 con 39,41\% de las referencias en esta subcategoría. Por otro lado, los docentes E2, E6, E7, E8, E9, E10, E11, E15 y E16, siendo los cuatro últimos pertenecientes a la escuela bilingüe para Sordos, no hicieron ninguna mención a esta subcategoría.

Los datos representan que los docentes de las escuelas inclusivas se refirieron menos a la comunicación directa con el alumno Sordo, representando la falta de conocimiento sobre la Libras, de estos, los entrevistados E3 y E5 no hicieron ninguna referencia, aunque el entrevistado E12, de la escuela bilingüe para Sordos también no haya mencionado esta subcategoría. De otro modo, el docente que más hizo referencia a la comunicación directa fue el E10, docente de la escuela bilingüe para Sordos, con 34,59\% de la referencias de esta subcategoría, de acuerdo con lo demostrado en las declaraciones de los entrevistados

Se nota que la hipótesis de que los docentes no utilizan el trabajo diferenciado con los alumnos Sordos porque no poseen una comunicación directa con ellos, pues no saben la Libras, fue comprobada por los datos y las declaraciones. Siguiendo esta lógica, el profesor que no tiene una comunicación directa con su alumno puede transferir, consecuentemente, la responsabilidad de su trabajo para otro profesional, en este caso el intérprete de Libras, como se muestra en las declaraciones del E8

Los docentes de las escuelas inclusivas se destacaron con relación a esta transferencia de responsabilidades de enseñanza para el profesional intérprete de Libras, y el entrevistado E3 fue el que más mencionó esta transferencia, con 81,06\%, seguido por E8, con 11,1\% de la referencia de esta subcategoría.

\section{Tabla 3}

Porcentaje de la declaración de los docentes con relación a las Metodologías de enseñanza de PL2

\begin{tabular}{|c|c|c|c|c|c|}
\hline $\begin{array}{l}\text { Partici- } \\
\text { pantes }\end{array}$ & $\begin{array}{l}\text { Estrategias de } \\
\text { Realización }\end{array}$ & $\begin{array}{l}\text { Acción-Reflexi- } \\
\text { ón-Reacción }\end{array}$ & $\begin{array}{c}\text { Ausencia de } \\
\text { Trabajo Dife- } \\
\text { renciado }\end{array}$ & $\begin{array}{c}\text { Comunicación } \\
\text { Directa }\end{array}$ & $\begin{array}{l}\text { Transferencia de Responsabi- } \\
\text { lidades para el Intérprete }\end{array}$ \\
\hline E2 & 5,22 & 9,03 & 0 & 12,55 & 0 \\
\hline E3 & 0,97 & 0 & 39,41 & 0 & 81,06 \\
\hline E4 & 6,09 & 5,43 & 4,88 & 9,65 & 0 \\
\hline E5 & 2,22 & 6,23 & 2,31 & 0 & 0 \\
\hline E6 & 5,55 & 3,92 & 0 & 0,72 & 0 \\
\hline E7 & 5,67 & 6,54 & 0 & 6,52 & 0 \\
\hline E8 & 2,88 & 2,48 & 0 & 5,15 & 11,1 \\
\hline E9 & 6,89 & 6,9 & 0 & 4,99 & 0 \\
\hline E10 & 10,81 & 7,65 & 0 & 34,59 & 0 \\
\hline E11 & 8,47 & 2,47 & 0 & 12,71 & 0 \\
\hline E12 & 5,84 & 6,15 & 31,13 & 0 & 6,63 \\
\hline E13 & 8,44 & 3,23 & 6,48 & 0,64 & 0 \\
\hline E14 & 13,09 & 24,66 & 6,03 & 2,33 & 1,21 \\
\hline
\end{tabular}




\begin{tabular}{c|c|c|c|c|c}
\hline $\begin{array}{c}\text { Partici- } \\
\text { pantes }\end{array}$ & $\begin{array}{c}\text { Estrategias de } \\
\text { Realización }\end{array}$ & $\begin{array}{c}\text { Acción-Reflexi- } \\
\text { ón-Reacción }\end{array}$ & $\begin{array}{c}\text { Ausencia de } \\
\text { Trabajo Dife- } \\
\text { renciado }\end{array}$ & $\begin{array}{c}\text { Comunicación } \\
\text { Directa }\end{array}$ & $\begin{array}{c}\text { Transferencia de Responsabi- } \\
\text { lidades para el Intérprete }\end{array}$ \\
\hline E15 & 3,59 & 4,76 & 0 & 2,65 & 0 \\
\hline E16 & 6,88 & 5,18 & 0 & 2,57 & 0 \\
\hline E17 & 7,38 & 5,38 & 9,76 & 4,91 & 0 \\
\hline
\end{tabular}

Nota. ${ }^{*}$ Los valores de porcentaje fueron calculados a partir da frecuencia del habla de cada entrevistado y de acuerdo con las categorías encontradas.

De acuerdo con la Tabla 4, se observa que los docentes de la escuela bilingüe para Sordos poseen más concienciación de la importancia de buenas prácticas pedagógicas relacionadas a la Lengua Portuguesa para Sordos, y su principal representante el entrevistado E15 con 19,99\% de las referencias de esta categoría. Se puede observar que los docentes de la escuela bilingüe para Sordos tuvieron mayor y menor representación por medio del E10 con 19,96\% de las referencias y a través del E13 con 0,94\%, analizándose por medio de las declaraciones de los entrevistados:

\section{Tabla 4}

Porcentaje de declaración de los docentes con relación a sus concepciones sobre la capacitación para actuación con alumnos Sordos

\begin{tabular}{c|c|c}
\hline Participante & $\begin{array}{c}\text { Concienciación de la Importancia de las } \\
\text { Buenas Prácticas }\end{array}$ & $\begin{array}{c}\text { Estrategias de Perfeccionamiento de las } \\
\text { Prácticas }\end{array}$ \\
\hline E2 & 17,41 & 3,6 \\
\hline E3 & 2,45 & 2,08 \\
\hline E4 & 6,46 & 9,39 \\
\hline E5 & 0 & 4,4 \\
\hline E6 & 0 & 7,06 \\
\hline E7 & 4,71 & 8,23 \\
\hline E8 & 1,94 & 2,51 \\
\hline E9 & 0 & 2,5 \\
\hline E10 & 0 & 19,96 \\
\hline E11 & 17,31 & 6,9 \\
\hline E12 & 2,26 & 2,03 \\
\hline E13 & 7,57 & 0,94 \\
\hline E14 & 8,45 & 9,4 \\
\hline E15 & 19,99 & 5,93 \\
\hline E16 & 3,65 & 13,46 \\
\hline E17 & 7,8 & 1,62 \\
\hline
\end{tabular}

Nota. ${ }^{*}$ Los valores de porcentaje fueron calculados a partir da frecuencia del habla de cada entrevistado y de acuerdo con las categorías encontradas.

Sobre la frecuencia de la codificación de las categorías por participante, de acuerdo con la Tabla 5, es relevante considerar que ninguno de los participantes se refirió a todas las 16 categorías presentadas en el árbol categorial. No obstante, el docente E14, de la escuela bilingüe para Sordos, fue el que se refirió a más categorías en su discurso, con 72 referencias en 12 
categorías diferentes, lo que indica que el discurso de este participante consiguió contemplar el mayor número de temática consideradas relevantes para la investigación.

Fue posible observar aún que los participantes E3 y E6, docentes de las escuelas inclusivas, fueron los entrevistados que se refirieron a menos categorías, con un total de 37 y 42 referencias, respectivamente, en tan solo siete categorías cada uno, lo que confirma que el discurso de estos participantes abordó menos temáticas entre las consideradas relevantes para los objetivos de la investigación.

Aunque la representación de mayor y menor número de categorías referenciadas sea cada una de un grupo de participantes, comparándose los dos grupos, docentes de las escuela inclusivas y docentes de la escuela bilingüe para Sordos, no fue posible verificar diferencias estadísticamente significativas, y esta comparación fue realizada a través del T-Test $(0,2416$, para $\mathrm{p} \leq 0.05$ ), o sea, los grupos no son estadísticamente distintos.

\section{Tabla 5}

Frecuencia de codificación de las categorias por participante

\begin{tabular}{c|c|c|c}
\hline Participante & Categorías & \% & Referencias* \\
\hline E2 & 11 & 68,75 & 53 \\
\hline E3 & 7 & 43,75 & 37 \\
\hline E4 & 11 & 68,75 & 46 \\
\hline E5 & 8 & 50 & 43 \\
\hline E6 & 7 & 43,75 & 42 \\
\hline E7 & 11 & 68,75 & 46 \\
\hline E8 & 10 & 62,5 & 41 \\
\hline E9 & 8 & 50 & 36 \\
\hline E10 & 8 & 50 & 46 \\
\hline E11 & 10 & 62,5 & 45 \\
\hline E12 & 10 & 62,5 & 30 \\
\hline E13 & 10 & 62,5 & 72 \\
\hline E14 & 12 & 75 & 41 \\
\hline E15 & 9 & 56,25 & 37 \\
\hline E16 & 9 & 56,25 & 50 \\
\hline E17 & 11 & 68,75 & \\
\hline T-Test & 0,2416 & 59,38 & \\
\hline p $\leq 0.05$ & & media & \\
\hline T16 & & & \\
\hline & & & \\
\hline
\end{tabular}

Nota. Utilizado el Test-T para comparación de las medias presentadas por los dos grupos.

Nivel de significancia $\mathrm{p} \leq 0.05$.

Los valores de porcentaje fueron calculados a partir da frecuencia del habla de cada entrevistado y de acuerdo con las categorías encontradas.

* Este valor se refiere al número de veces que cada participante se refirió a cada categoría.

\section{Discusión}

Se puede inferir que la capacitación inicial de los profesores investigados está de acuerdo a lo que expone la Ley de Directrices y Bases de la Educación Nacional (LDBEN) en lo que dice respecto al grado de estudios, o sea, todos con nivel superior completo para el 
trabajo en la educación básica (Ley no 9.394, 1996). No obstante, no significa que esta graduación haya contemplado todas las áreas de conocimiento necesarias a las actividades docentes, principalmente con relación a la educación de Sordos.

Bajo este prisma de la capacitación inicial en la perspectiva de la educación de Sordos, se observó que tres de los 16 participantes se graduaron después de la promulgación del Decreto Federal no 5626 de 22 de diciembre de 2005, lo que hizo obligatoria la inserción de la Unidad Curricular (UC) Libras en los cursos de licenciatura y Fonoaudiología (Decreto no 5.626, 2005). Sin embargo, ninguno de los docentes entrevistados cursó esta UC en su periodo de graduación.

Los datos presentados aquí no permitieron inferir sobre los beneficios de la inserción de la UC Libras en los cursos de licenciatura, pero Santos e Klein (2015) pudieron deducir que todos los trabajos indican contribuciones que la asignatura de Libras proporciona a la formación de los profesores, del mismo modo como a la promoción de la inclusión de los alumnos Sordos.

Específicamente cuanto a la formación en Lengua de Señales, Pagnez (2016) retrata datos que se asocian a la importancia de que la UC de Libras sea inserida en el periodo de capacitación inicial; la autora que analizó la mediación profesor y alumno en la consolidación de conocimientos sobre la Educación Especial y de Libras en un curso semipresencial para capacitación de profesores que actuaron en el área de la Enseñanza de Ciencias, constató que el prejuicio y la discriminación que no eran reconocidos por los alumnos entre sí como en la sociedad se hicieron evidentes en la convivencia en la asignatura y con la posibilidad de discusión y reflexión sobre las prácticas, además de que $18,51 \%$ de los alumnos del curso eligen realizar los trabajos de conclusión de curso en el área de inclusión con el objetivo de profundizar los conocimientos y favorecer la actuación pedagógica.

De acuerdo con la base legal de la formación de docentes en la perspectiva de la Educación Especial Inclusiva (Ley no 9.394, 1996; Ley no 13.005, 2014), se espera que los docentes en formación tengan contacto con la Lengua de Señas en sus cursos de graduación. Es bajo este aspecto que Louzada et al. (2015) tras analizar los planos de enseńanza de los cursos de licenciatura en las áreas de Ciencias Humanas, Biológicas y Exactas, realizando análisis de contenido a partir de los temas propuestos en cuestiones abiertas concluyeron que hay mayor incidencia de asignaturas asociadas a la educación en el área de humanidades, especialmente la de Lengua Brasileña de Señas (Libras).

En comparación de la formación inicial de Brasil y Argentina, bajo el aspecto de la actuación con alumnos Sordos, la Resolución no 5296/08 de Argentina postula nuevos Diseños Curriculares a la enseńanza superior y propone la inclusión de talleres de Lengua de Señales Argentina (LSA) como instancia curricular dentro de la carrera, aumentando el contacto de los docentes con la lengua y cultura Sordas (Sago, 2015). Mientras que la legislación brasileña plantea también la unidad curricular de Lengua de Señas Brasileña, siguiendo una mirada para la inclusión efectiva.

La falta de conocimiento de la estructura lingüística de la Lengua de Señas y de la cultura Sorda por el profesor oyente que actúa con alumnos Sordos es un obstáculo en el proceso de enseñanza de la escrita en Lengua Portuguesa (Castro \& Calixto, 2016). 
Se puede entender que la capacitación inicial de los profesores investigados careció de UC's específicas sobre la educación de Sordos, lo que no contempla aspectos culturales, lingüísticos o discusiones acerca de las estrategias de aprendizaje de este público. Para esto, se analizó el ítem capacitación continua bajo el mismo prisma de la educación de Sordos.

Bajo el aspecto de la formación continua, fue posible observar que 13 profesores estudiaron postgrado, tanto en la modalidad Lato Sensu como Stricto Sensu, comprendiéndose que el perfil encontrado en la investigación contrapone los datos del perfil de los profesores brasileños, una vez que 83,9\% de los profesores de la educación básica brasileña posee solamente graduación (Departamento Intersindical de Estatística e Estudos Socioeconômicos [DIEESE], 2014).

Aunque los datos de la presente investigación no estén de acuerdo con los estudios sobre la capacitación continua, en el sentido de que los docentes entrevistados poseen una capacitación más allá del perfil de los profesores brasileños, los docentes aún necesitan de la oferta de cursos de postgrado, principalmente en el área Educación Especial, Inclusiva y Educación de Sordos, puesto que algunas de las competencias que antes eran de dominio exclusivo de los profesores de Educación Especial o de las escuelas especiales "migraron" para las escuelas y profesores reglados (Hoppey et al., 2004).

Los datos encontrados se justifican por la gran implementación y reformulación de los planes de carrera de los docentes en la última década con la utilización de la titulación académica como uno de los criterios para evolución del profesional, como previsto en el artículo 67 de la LDBEN, que trata de la valorización de los profesionales de la educación a través del perfeccionamiento profesional continuo (Ley no 9.394, 1996).

De los docentes entrevistados, tres poseen el título de Magíster y cuatro el título de Doctor, y todos son de la escuela bilingüe para Sordos. Este perfil, también contrapone el perfil de los docentes brasileños de la educación pública, ya que solamente 4,5\% de los profesores poseen alguna de estas titulaciones (DIEESE, 2014). Este hecho puede justificarse aún por las exigencias del concurso público para ingreso en la carrera de este segmento que exige la titulación mínima de Magíster.

Aún en lo referente a la capacitación continua, más específicamente en relación con la actuación con los alumnos Sordos, se observó que cuatro de los docentes de las escuelas inclusivas juzgaron no poseer ningún nivel de conocimiento en Libras, a diferencia de los datos de la escuela bilingüe para Sordos donde todos los docentes apuntaron tener por lo menos el nivel básico de conocimiento en esta lengua. Estos datos se hacen evidentes por el hecho de que la institución de educación bilingüe para Sordos ofrece a los docentes cursos de Libras y capacitaciones relacionadas a la educación de Sordos, antes del inicio efectivo del trabajo, como requisito para la actuación docente.

En lo que dice respecto a las capacitaciones ofrecidas por la red federal en la escuela bilingüe para Sordos, esta fue relatada por todos los docentes de esta red como de gran importancia en la práctica con este tipo de alumno, por haber proporcionado subsidio para el trabajo del docente.

La falta de capacitación de profesores en la actuación con el alumnos Sordo provoca consecuencias directas en su aprendizaje, una vez que el profesor cae en el proceso de simpli- 
ficación en la construcción del conocimiento, precarizando la enseñanza y convirtiendo en chatarras las estrategias posibles.

Se percibe, por lo tanto, que la capacitación docente, específicamente de los profesores de Lengua portuguesa en la actuación con los alumnos Sordos es muy importante en el proceso de enseńanza, hecho comprobado por Tsukamoto (2014) el cual aclara que el gran fracaso en la enseñanza de la escritura y de la lectura en Lengua Portuguesa de los alumnos Sordos se debe a que la mayoría de los alumnos no tienen interese y gusto por aprender, ya que los profesores no están preparado para tal situación.

Estas estrategias específicas de enseñanza solo son construidas por el profesor a partir del momento en que este obtiene conocimiento sobre las especificidades de su alumno.

Estos conocimientos son aprendidos, en su mayoría, en el ejercicio de la actividad laboral, o sea, a través de una formación continua, no permitiéndose parar el sistema educativo para que se capaciten. En este sentido, la estrategia del poder público actual es la inversión en formación continua a través de cursos a nivel de perfeccionamiento y especialización en la modalidad a distancia por medio de la Universidad Abierta de Brasil (UAB) y en la modalidad presencial y semipresencial por la Red Nacional de Formación Continua de Profesores en la Educación Básica (Renafor) en asociación con Instituciones Públicas de Enseñanza Superior (IPES) (Victor \& Piloto, 2016).

En el aspecto de la formación en el trabajo, los datos de la investigación permiten comprender que la segunda forma más utilizada para la obtención del conocimiento por los profesores para actuación con alumnos Sordos fueron los cursos libres.

En este prisma, queda claro que los cursos libres son una estrategia posible y razonable en la inversión pública para la capacitación de profesores en la actuación con alumnos Sordos.

Aunque la obtención de conocimiento por parte de los profesores en la actuación con alumnos Sordos pueda ser de manera formal, a través de la capacitación inicial, continua y en cursos libres, el modo empírico ha demostrado que todavía es más utilizada por los docentes para la formación en Educación Especial.

Los datos de la investigación demostraron que todos los docentes mencionaron haber obtenido algún tipo de conocimiento sobre la educación de Sordos a través del contacto con los propios alumnos, con el profesional traductor-intérprete de Libras (TIL), con los colegas más experimentados y por medio de la búsqueda personal por materiales teóricos.

$\mathrm{Al}$ contrario de las estrategias utilizadas por los profesores de las escuelas inclusivas, los de la escuela bilingüe para Sordos, informaron la necesidad de recurrir a la enseńanza de Libras a los alumnos antes de empezar la enseñanza de la Lengua Portuguesa escrita. Este hecho ocurre porque la mayoría de los Sordos inscriptos en la escuela son hijos de padres oyentes, que inician el proceso de escolarización sin la Lengua de Señas adquirida.

Estos datos pueden ser confirmados por el estudio de Silva e Silva (2016) que buscaron comprender lo que los profesionales de la escuela dicen sobre el papel de la Libras en la educación de Sordos. Concluyen, así, que la mayoría de los Sordos en edad escolar no posee la 
Libras como primera lengua, pues 95\% de los Sordos son hijos de padres oyentes que desconocen la Lengua de Señas (Sacks, 2010).

En el aspecto de la comunicación de los docentes con los alumnos Sordos y las estrategias por ellos utilizadas, se comprende que los profesores de las escuelas inclusivas se destacan en la categoría de transferencia de responsabilidad para el traductor-intérprete de Libras y en la falta de comunicación directa con el alumno Sordo.

Los resultados de esta investigación, relacionados a la transferencia del trabajo del profesor para el traductor-intérprete de Libras, corroboran con la investigación de Vargas e Gobara (2014) la cual presenta un análisis de las interacciones entre el alumno con sordera, el profesor y el intérprete en clase, además del papel e estos sujetos en el proceso de inclusión del alumnos Sordos. Los autores evidenciaron que solo el intérprete interactuaba efectivamente con los alumnos y poco contribuya para que ellos interactuasen con personas que no dominan la Lengua Brasileña de Señas.

Este dominio de Lengua de Señas por los profesores posee relación directa con las estrategias pedagógicas específicas utilizadas por los docentes en la actuación con el alumno Sordo. En este sentido, los docentes de la escuela bilingüe informaron que utilizan el metalenguaje como recurso pedagógico en la enseñanza de portugués, o sea, la utilización de un lenguaje para describir otro lenguaje, como la utilización de la Libras para describir, reflexionar y comprender la propia Libras, inicialmente y después de la utilización de la Lengua Portuguesa escrita. Ya los profesores de las escuelas inclusivas no hacen uso del metalenguaje, puesto que no se comunican de manera directa con sus alumnos, utilizando el propio lenguaje para describirla.

Además de los recursos y estrategias visuales, los profesores, principalmente los de las escuelas inclusivas, cuentan con el apoyo diario del traductor intérprete de Libras (TIL) en sus clases, haciéndose un aliado en los momentos de dudas sobre cómo actuar con el público Sordo, ya que es el profesional especializado. Lacerda et al. (2014) afirman que para desarrollar prácticas académicas accesibles para el alumno Sordo, es necesario, antes de cualquier adaptación curricular, que haya unión entre profesor y traductor-intérprete de Libras.

Al cuestionarlos sobre la relación con el TIL, aunque estos no estén tan presentes en las clases de la escuela bilingüe, los docentes fueron unánimes y explicaron que no hay esta alianza en el planeamiento de las actividades y en el acceso previo a los contenidos por el TIL.

Se deduce, por tanto, que la unión y la buena relación entre el profesor regente y el profesional traductor-intérprete de Libras en la participación de este último en el planeamiento de las actividades y en el acceso con antelación de los contenidos que serán ministrados, ofrece, además del aprendizaje no formal, una reflexión sobre las estrategias de enseńanza que se utilizarán.

Este perfil reflexivo de los docentes entrevistados está de acuerdo con el perfil esperado por las bases legales para formación de profesores en Brasil, como las Directrices Curriculares Nacionales para la Formación de Profesores de Educación Básica, a nivel superior (Ley no 10.436, 2002), y las Directrices Curriculares Nacionales para Formación de Docentes de Educación Infantil y de los años iniciales de la Enseñanza Fundamental, a nivel medio (Resolución CEB no 2, 1999). En estos documentos son encontradas expresiones como "acción-reflexión-acción" como estrategia didáctica privilegiada para resolución de situaciones problema (Resolución CNE/ 
CP no 1, 2002), y "una concepción de profesor reflexivo" (Resolución CEB n² 2, 1999) necesaria a los cuestionamientos, pensamiento autónomo y ético con relación a las intervenciones en el ejercicio profesional que es práctica y contextualizada (Fagundes, 2016).

En sentido de "reelaborarse", los profesores mencionaron que las estrategias de enseñanza de Lengua Portuguesa para alumnos Sordos son basadas todavía en las actitudes de intento, error, acierto, y se hace necesario para tanto, la acción-reflexión-reacción de sus prácticas pedagógicas.

Bajo esta misma perspectiva reflexiva, los docentes fueron cuestionados sobre su opinión o experiencia vivida a propósito de las buenas prácticas adoptadas para su formación en lo que dice respecto a la actuación con el alumno Sordo, y aún sugerencias de mejorías en la formación de os futuros docentes.

En su totalidad, los docentes están de acuerdo con que la inserción de la Libras como Unidad Curricular en los cursos de licenciatura puede empoderar el profesor en la actuación con el alumno Sordo en clase. Sugiriendo que se impartiesen dos o más semestres de Libras con la intención de profundizar los conocimientos sobre la sordez, la educación de Sordos, los aspectos metodológicos y estrategias de enseńanza de portugués para Sordos.

Bajo esta misma perspectiva reflexiva, los docentes fueron cuestionados sobre su opinión o experiencia vivida a propósito de las buenas prácticas adoptadas para su formación en lo que dice respecto a la actuación con el alumno Sordo, y aún sugerencias de mejorías en la formación de os futuros docentes.

Esta construcción de conocimiento se encuentra con otro aspecto resaltado por los docentes entrevistados, la obtención de conocimiento no formal, o sea, con el fin de compartir informaciones en el intento de generar una red de enseńanza colaborativa con los profesionales específicos del área de la sordez, como los traductores intérpretes de Lengua de Señas y profesores más experimentados en el área de actuación.

\section{Conclusiones}

Fue posible percibir, en el caso específico de la presente investigación, que la formación de los profesores posee una influencia directa en sus prácticas en clase en la enseńanza de Lengua Portuguesa para Sordos. Se resaltó la dicotomía entre los de las escuelas inclusivas y los de la escuela bilingüe para Sordos, en lo que dice respecto al locus de la lengua de la instrucción, o sea, los profesores de las escuelas inclusivas utilizan materiales visuales, Power Point, adaptación de textos escritos, pruebas y actividades configurando un vocabulario más sencillo, sin diálogo y reflexionando con el contenido en Libras, convirtiéndola en una lengua accesoria.

Por otro lado, los docentes de la escuela bilingüe, trabajaban desde el punto de vista de la Libras como Lengua de instrucción, utilizando el metalenguaje para la enseñanza de portugués como segunda lengua, además de utilizar el recuento en Libras, materiales visuales y recursos con imágenes, estos últimos declaran conseguir éxito en las estrategias utilizadas y los primeros no. 
A partir de las proposiciones mencionadas por los docentes, se espera contribuir de manera significativa para la formación de futuros profesionales de la educación que deseen trabajar con alumnos Sordos, además de incentivar algunas ideas posibles de ser puestas en práctica por los poderes públicos con la inversión en capacitación inicial y continua de los profesores. Se resalta que esta inversión no es necesariamente financiera, pues no hay nadie más capacitado para formar otros profesionales que los iguales.

\section{REFERÊNCIAS}

Bardin, L. (2011). Análise de Conteúdo. Ediçôes 70.

Bernieri-Souza, R., \& Vasconcelos, C. E. B. (2021). Ensino e aprendizagem de português como segunda língua para surdos, Revista Trem de Letras, 8(1), 1-24.

Brochado, S. M. D. (2003). A apropriação da escrita por crianças surdas usuárias da língua de sinais brasileira [Tese de Doutorado, Universidade Estadual Júlio de Mesquita Filho - UNESP]. Repositório da UNESP. https://repositorio.unesp.br/handle/11449/102476

Calixto, H. R. S., Ribeiro, A. E. A., \& Ribeiro, A. A. (2019). Ensino de língua portuguesa escrita na educação bilíngue de surdos: questóes a partir de narrativas de professores da Baixada Fluminense. Revista Brasileira de Estudos Pedagógicos, 100(256), 578-593. https://doi.org/10.24109/2176-6681. rbep.100i256.4021

Castro, F. G. A. S., \& Calixto, H. R. S. (2016). Português para surdos e as tecnologias digitais. Journal of Research in Special Educational Needs, 16(1), 870-875. https://doi.org/10.1111/1471-3802.12344

Decreto $n^{\circ}$ 5.626, de 22 de diciembre de 2005. (2005). Regula a Lei no 10.436, de 24 de abril de 2002, que dispóe sobre a Língua Brasileira de Sinais - Libras, e art. 18 da Lei no 10.098, de 19 de dezembro de 2000. http://www.planalto.gov.br/ccivil_03/_ato2004-2006/2005/decreto/d5626. htm\#: : :text=DECRETO\%20N\%C2\%BA\%205.626\%2C\%20DE\%2022,19\%20de\%20 dezembro\%20de\%202000.

Departamento Intersindical de Estatística e Estudos Socioeconômicos. Nota Técnica. (2014). Transformaçôes recentes no perfil do docente das escolas estaduais e municipais de educaçâo básica. DIEESE.

Fagundes, T. B. (2016). Os conceitos de professor pesquisador e professor reflexivo: perspectivas do trabalho docente. Revista Brasileira de Educação, 21(65), 281-298. https://doi.org/10.1590/S141324782016216516

Hoppey, D., Yendol-Silva, D., \& Pullen, P. C. (2004). We became teachers together: Understanding collaborative teaching as innovation in teacher education. Action in Teacher Education, 26(1), $12-$ 26. https://doi.org/10.1080/01626620.2004.10463309

Kumada, K. M. O., \& Prieto, R. G. (2019). Desdobramentos da política de educação superior para formação do docente de libras. Cadernos de Pesquisa, 49(173), 64-84. https://doi. org/10.1590/198053145975

Lacerda, C. B. F., Santos, L. F., \& Caetano, J. F. (2014). Estratégias metodológicas para o ensino de alunos surdos. In C. B. F. Lacerda, \& L. F. Santos (Eds.), Tenho um aluno surdo, e agora? Introdução à Libras e educação de surdos (pp. 185-200). EdUFSCar.

Lage, M. C. (2011). Utilização do software NVivo em pesquisa qualitativa: uma experiência em EaD. ETD - Educ. Tem. Dig., 12(n.esp.), 198-226. https://doi.org/10.20396/etd.v12i0.1210 
Lei no 9.394, de 20 de dezembro de 1996. Institui a Lei de Diretrizes e Bases da Educação Nacional. http://www.planalto.gov.br/ccivil_03/leis/19394.htm

Lei no 10.436, de 24 de abril de 2002. Dispóe sobre a Língua Brasileira de Sinais e dá outras providências. http://www.planalto.gov.br/ccivil_03/leis/2002/110436.htm

Lei no 13.005, de 25 de junho de 2014. Aprova o Plano Nacional de Educaçáo e de outras providencias. http://www.planalto.gov.br/ccivil_03/_ato2011-2014/2014/lei/113005.htm\#:-:text=Aprova\%20 o\%20Plano\%20Nacional\%20de,Art.

Lei $n^{o}$. 13.146, de 6 de julho de 2015. Institui a Lei Brasileira de Inclusão da Pessoa com Deficiência (Estatuto da Pessoa com Deficiência). http://www.planalto.gov.br/ccivil_03/_ato2015-2018/2015/ lei/113146.htm.

Louzada, C. A., Martins, S. E. S. O., \& Giroto, C. R. M. (2015). Formación de profesores en la perspectiva de la educación inclusiva en Brasil. Revista Mexicana de Investigación Educativa, 20(64), 95-122. http://www.scielo.org.mx/scielo.php?script=sci_arttext\&pid=S1405-66662015000100006

Morais, M. P., \& Martins, V. R. O. (2020). Educação bilíngue inclusiva para surdos como espaço de resistência. Pro-Posiçôes, 31(e20180089). https://dx.doi.org/10.1590/1980-6248-2018-0089.

Oliveira, J. B. G. (2018). Educação de surdos e o português como segunda língua: reflexôes e uma experiência de ensino. Convenit Internacional 27, Cemoroc-Feusp / IJI - Univ. do Porto, 97-104.

Pagnez, K. S. M. M. (2016). A formação de professores para atuar na inclusão escolar. Journal of Research in Special Educational Needs, 16(1), 70-74. https://doi.org/10.1111/1471-3802.12127

Quadros, R. M., \& Schmiedt, M. L. P. (2006). Ideias para ensinar português para alunos surdos. MEC, SEESP.

Resolução CEB no 2, de 19 de abril de 1999. Institui Diretrizes Curriculares Nacionais para a Formação de Docentes da Educaçáo Infantil e dos anos iniciais do Ensino Fundamental, em nível médio, na modalidade Normal. http://portal.mec.gov.br/escola-de-gestores-da-educacao-basica/323secretarias-112877938/orgaos-vinculados-82187207/13203-resolucao-ceb-1999 .

Resolução CNE/CP no 1, de 18 de fevereiro de 2002. Institui Diretrizes Curriculares Nacionais para a Formação de Professores da Educação Básica, em nível superior, curso de licenciatura, de graduação plena. http://portal.mec.gov.br/escola-de-gestores-da-educacao-basica/323-secretarias-112877938/ orgaos-vinculados-82187207/13207-resolucao-cp-2002 .

Resolución no 5.296 de 2008. Institui Maestro Especial de Lengua de Señas Argentina -LSA. https://abc. gob.ar/especial/sites/default/files/2009_-_documento_de_apoyo_-_planificacion_linguistica.pdf.

Sacks, O. (2010). Vendo vozes: uma viagem ao mundo dos surdos. Companhia de Bolso.

Sánchez, C. (1990). La increíble y triste historia de la sordera. CEPROSORD.

Sago, M. (2015). La formación de profesores para el abordaje de la deficiencia auditiva en el GCBA. In Buenos Aires. Una mirada transversal de la sordera (pp. 19-22). Comisión para la Plena Participación e Inclusión de las Personas con Discapacidad - COPIDIS.

Santos, A. N., \& Klein, M. (2015). Disciplina de Libras: o que as pesquisas acadêmicas dizem sobre a sua inserção no ensino superior. Revista Reflexão e Ação, 23(3), 9-29. https://doi.org/10.17058/rea. v23i3.6147

Silva, C. M., \& Silva, D. N. H. (2016). Libras na educação de surdos: o que dizem os profissionais da escola. Psicologia escolar e educacional, 20(1), 33-43. https://doi.org/10.1590/2175-353920150201917 
Silva, G. M. (2017). O português como segunda língua dos surdos brasileiros: uma apresentação panorâmica. Revista X, 12(2), 130-150. https://doi.org/10.5380/rvx.v12i2.51140

Tsukamoto, N. M. S. (2014). A vereda histórica da educação dos surdos: da oralidade ao bilinguismo na ótica da formação docente. In S. Andreis-Witkoski, \& M. R. P. Filietaz (Orgs.), Educação de Surdos em Debate (pp. 247-262). Editora UTFPR.

Vargas, J. S., \& Gobara, S. T. (2014). Interações entre o aluno com surdez, o professor e o intérprete em aulas de Física: uma perspectiva Vygotskiana. Revista Brasileira Educação Especial, 20(3), 449-460. https://doi.org/10.1590/S1413-65382014000300010

Victor, S. L., \& Piloto, S. S. F. H. (2016). Formação do professor no contexto do Observatório Nacional de Educação Especial. In S. L. Oliveira, \& I. M. Oliveira (eds.), Educação Especial: políticas e formação de professores(pp. 159-178). ABPEE.

Vieira, C. R., \& Molina, K. S. M. (2018). Prática pedagógica na educação de surdos: o entrelaçamento das abordagens no contexto escolar. Educação e Pesquisa, 44(e179339). https://doi.org/10.1590/ s1678-4634201844179339

Recebido em: 12/01/2021

Reformulado em: 16/04/2021

Aprovado em: 21/04/2021 
SOARES, R.S.M.V. et al. 